\title{
A Novel Member of the Subtilisin-Like Protease Family from
}

\section{Bacillus pumilus 7P: Properties and Applications}

\author{
Sharipova $\mathbf{M}^{1 *}$ and Mardanova $\mathbf{A}^{2}$ \\ 1Professor, Kazan Federal University, Russia \\ ${ }^{2}$ Leading Researcher, PhD, Kazan Federal University, Russia
}

*Corresponding author: Professor Margarita Sharipova, Department of Microbiology,

\section{Mini Review}

Volume 4 Issue 3

Received Date: November 28, 2019

Published Date: December 10, 2019

DOI: $10.23880 /$ oajmb-16000152

Kazan Federal University, 420008, Kazan, Kremlevskaya str., 18, Russia, Tel: +7(843)2337856; Email: marsharipova@gmail.com

\section{Abstract}

AprBp is a $1146 \mathrm{bp}$ gene of Bacillus pumilus strain 7P that encodes an extracellular serine proteinase, characterized by significant similarity with subtilisins. The start sites of transcription (T) and translation (GTG) of the gene have been identified. A regulatory network for controlling proteinase gene expression has been established. Proteinase AprBp was isolated from the culture fluid at different growth phases, and the primary structure of the protein was identified at the variuos phases of growth. In the stationary phase, the enzyme forms dimers and is very stable. A feed additive based on B. pumilus proteinase is being developed for poultry farming.

Keywords: Serine Proteinase; Microorganism; Bacillus pumilus; Feed additive

\section{Introduction}

Serine proteases perform a diverse array of physiological and cellular function, spanning from digestive and degradative processes to regulatory protein processing, cellular immunity, remodeling cell population and fibrinolysis. Currently, more than 200 proteases of both bacterial and higher eukaryotic origin have been ascribed to the superfamily of subtilases (subtilisin-like serine proteases) [1]. With recent advancements in sequencing technology, the number of new members of the subtilases family is surging. This review presents information about a new proteinase from $\mathrm{B}$. pumilus $7 \mathrm{P}$ with high similarity to subtilisins from other Bacillus species.

The B. pumilus 7P whole-genome sequence and annotation data have been deposited at
DDBJ/EMBL/GenBank under the accession no. JHUD00000000 [2]. This strain is widely used in studying enzymes of interest for industrial utilization. A dendrogram analysis based on alignment of the catalytic domains using the MEROPS database (version 10.0) revealed $153 \mathrm{ORF}$ for proteases, 60 of which belong to serine proteases [3]. Nucleotide sequence analysis of the region, part of the EMBL accession number AY754946, revealed an $1146 \mathrm{bp}$ ORF (aprBp) that encodes an extracellular serine proteinase. Transcription analysis, performed by RT-PCR and primer extension, allowed the localization of transcription start site (T). To identify the translation start, the modified of aprBp with mutation in putative start codons were developed and it was concluded that the translation in aprBp gene starts from GTG codon [4]. It was uncovered that complete expression of the proteinase gene requires an optimal promoter with a length of $445 \mathrm{bp}$. Fusion constructions 


\section{Open Access Journal of Microbiology \& Biotechnology}

with reporter protein gfp and gel retardation assay indicated that, regulatory proteins DegU-P and Spo0A were involved in the binding complex with promotor of aprBp. Using regulatory protein mutants showed differences in the regulatory network of proteinase expression at different stages of bacterial growth [5].

At the stage of vegetative growth, proteinase expression was regulated by catabolite repression and the regulatory system DegU/DegS, while during sporulation its expression is controlled by spo-genes [5]. Inactivation of the aprBp gene in the genome leads to a pleotropic effect, the strain's resistance to stresses decreases, the spectrum of extracellular proteins and some biochemical and morphological characteristics change. The expression of the aprBp gene was developed according to the twostage mechanism during vegetative growth and at the stage of spore formation. We isolated proteinase from the culture fluid of B. pumilus at different growth stages: $24 \mathrm{~h}$ (early proteinase) and $48 \mathrm{~h}$ (late proteinase). MALDI-TOF mass spectrometry of both purified enzymes demonstrated that they were identical amino acid sequence with equal $\mathrm{N}$ - and $\mathrm{C}$ - ends [6]. The molecular weights of both proteinases were $27 \mathrm{kDa}$ and their isoelectric points were 6.64. This value is lower than that of other subtilases (pI 7.8 for BPN'and pI 9.4 for Carlsberg). The substrate specificity of proteins was studied on synthetic peptides [7]. Enzymes do not cleave substrates specific for chymotrypsin (Glp-Phe-Gly-pNa), Glu-Asp-specific proteases (Z-Glu-pNa), but both are active against substrates for subtilisins (Glp-Ala-Ala-LeupNa, Z-Ala-Ala-Leu-pNa). Both enzymes just like most subtilisin-like serine enzymes (EC 3.4.21.62) effectively hydrolyze n-nitroanilides containing hydrophobic amino acids (Leu and Phe) [7]. Biochemical analysis revealed differences in $\mathrm{Km}$ values for proteinase isolated at different growth stages $(1.85 \mathrm{mM}$ and $0.86 \mathrm{mM}$ for the early and late one respectively) [6]. Thus, the enzyme in stationary stage has a high affinity for substrates and catalyzes the cleavage reaction more efficiently. Our data also confirm the results of hydrolysis of the insulin Bchain, the late enzyme hydrolyzes insulin B chain more effectively, in comparison to the early protein [7]. Both enzymes are not inhibited by natural inhibitors, such as ovomucoid, anemone inhibitor, and trypsin inhibitor [7]. Both proteins are inhibited by a specific inhibitor of serine proteinases PMSF and are not inhibited by inhibitors of metalloproteinases, o-phenantroline and EDTA. The activity of early enzyme was maximal at $37^{\circ} \mathrm{C}$ and $\mathrm{pH} 10.0$, while that of the late enzyme was at $45^{\circ} \mathrm{C}$ and $\mathrm{pH}$ 10.0. The enzyme was stable within a $\mathrm{pH}$ range of 6.5 to 11.5 and remains active in the temperature range of $0-55^{\circ} \mathrm{C}$. The addition of calcium ions increased the activity of the enzyme of both stages of growth by an average of $20-70 \%$. It was found that the proteinase forms dimers with the participation of calcium ions in the stationary phase and is characterized by increased stability [6]. Proteinase of both stages of growth is a product of one gene, the mechanism of regulation of expression of which differs depending on the phase of growth of bacteria [5].

We studied the effect of gastrointestinal secretions on protease activity. The enzyme retained up to $60 \%$ of activity in the presence of gastric juice of chickens $(\mathrm{pH}$ of 3 ). In the presence of pancreatic and intestinal juice, proteinase activity was fully preserved. At bile concentrations from $0.01 \%$ to $0.05 \%$ for $1 \mathrm{~h}$, proteinase activity remained at the control level. With an increase in concentration to $1 \%$, a $10 \%$ decrease in enzyme activity was observed. Based on the study of the properties of the enzyme, we concluded that they can serve as potential feed additives for birds. Using the $\mathrm{B}$. pumilus 7P proteinase, we are in the process of developing a food supplement for poultry farming. In preliminary tests, the addition of proteinases to the diet of birds led to an increase in bird productivity and had a positive effect on the uptake of nitrogen-containing compounds.

\section{Acknowledgement}

This work was supported by the Russian Science Foundation [project no.16-16-04062] and performed in accordance with the Russian Government Program of Competitive Growth of Kazan Federal University.

\section{References}

1. Siezen RJ, Leunissen JA (1997) Subtilases: the superfamily of subtilisin-like serine proteases. Protein Sci 6(3): 501-523.

2. Elena I Shagimardanova, Anna A Toymentseva, Nelly P Balaban, Ayaslu M Mardanova, Yulia V Danilova, et al. (2014) Draft Genome Sequence of Bacillus pumilus 7P, Isolated from the Soil of the Tatarstan Republic, Russia. Genome Announc 2(3): e00599-14.

3. Pudova DS, Toimentseva AA, Sharipova MR (2018) Bacillus pumilus proteases and protease inhibitors. Modern Science International Scientific Journal 11: 47-54. 
4. Kayumov AR, Sabirova AR, Balaban NP, Mardanova AM, Ilinskaya ON, et al. (2008) Start codon in the Bacillus intermedius gene for serine proteinase. Molecular Biology 42(1): 105-109.

5. Sharipova M, Balaban N, Kayumov A, Kirillova Y, Mardanova A, et al. (2008) Tre expression of the serine proteinase gene of Bacillus intermedius in Bacillus subtilis. Microbiological Research 163(1): 3950.

6. Ekaterina O Mikhailova, Nelly P Balaban, Ayslu M Mardanova, Nataliya L Rudakova, Olga N Ilyinskaya, et al. (2009) Purification of a subtilisin-like serine proteinase from recombinant Bacillus subtilis during different phases of growth. Annals of Microbiology 59(2): 301-307.

7. Mikhailova EO, Mardanova AM, Balaban NP, Rudenskaya GN, Sharipova MR (2007) Isolation and characterization of a subtilisin-like proteinase of Bacillus intermedius secreted by the Bacillus subtilis recombinant strain AJ73 at different growth stages. Biochemistry (Moscow) 72(2): 192-198. 